\title{
Variação das características físicas da madeira de Eugenia rostrifolia
}

Luana Candaten $\oplus^{1 *}$ Tauana de Souza Mangini $\oplus^{2}$ Eduarda Bandera $\oplus^{2}$ Laura da Silva Zanchetta $\oplus^{2}$ Rômulo Trevisan $\odot^{2}$ Jonathan William Trautenmuller $\circledast^{3}$

${ }^{1}$ Universidade de São Paulo, Av. Pádua Dias - Agronomia, Piracicaba - SP, 13418-900, Piracicaba, SP, Brasil

${ }^{2}$ Universidade Federal de Santa Maria, Campus Frederico Westphalen, Linha Sete de Setembro, s/n, CEP 98400-000, Frederico Westphalen, RS, Brasil

${ }^{3}$ Universidade Federal do Paraná, Av. Pref. Lothário Meissner, 632, Jardim Botânico, Campus III, CEP 80210-170, Curitiba, PR, Brasil

\section{Original Article \\ *Corresponding author:}

lcandaten@usp.br

Palavras-chave:

Propriedades físicas

Qualidade da madeira

Espécie nativa

Keywords:

Physical properties

Wood quality

Native species

Received in

2020/03/16

Accepted on

2020/04/28

Published in

2020/06/30

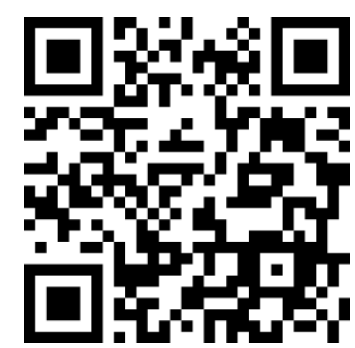

DOI:

https://doi.org/10.34062/afs. v7i2.10017

\section{(cc) BY}

RESUMO: O objetivo do estudo foi verificar a variação radial e longitudinal, da massa específica básica, aparente saturada e do teor de umidade no fuste de Eugenia rostrifolia. No experimento foram utilizadas três árvores de Eugenia rostrifolia para determinação das características físicas de acordo com as recomendações da norma ASTM D-143-94 (1995). Os dados foram analisados estatisticamente por meio do procedimento Stepweise e variável Dummy, ambos a partir de modelos de regressão, com o intuito de representar múltiplas variáveis qualitativas. Os resultados mostraram um decréscimo dos valores de massa específica básica e aparente saturada da base para o topo da árvore, bem como da medula até a casca. Já o teor de umidade não apresentou alterações estatísticas nos planos analisados. Os modelos obtidos para descrever a massa específica básica e a saturada no sentido radial, apesar de apresentarem tendência de decréscimo, não podem ser utilizados para todas as posições axiais pois, de acordo com a variável Dummy analisada, evidenciaram padrões decrescentes distintos. A madeira de Eugenia rostrifolia pode ser classificada como moderadamente pesada de acordo com o valor de massa específica básica igual a $0,694 \mathrm{~g} \mathrm{~cm}^{-3}$. As características físicas obtidas demonstraram que a espécie tem potencial para uso em vários segmentos como, por exemplo, construção civil e movelaria.

\section{Variation in physical characteristics of Eugenia rostrifolia wood}

\begin{abstract}
The objective of the study was to verify the variation, radial and longitudinal, of the basic density, saturated apparent specific mass and the moisture content in the Eugenia rostrifolia stem. In the experiment, three Eugenia rostrifolia trees were used to determine the physical characteristics according to the recommendations of ASTM D-143-94 (1995). The data were statistically analyzed by Stepweise procedure and Dummy variable, both from regression models, in order to represent multiple qualitative variables. The results demonstrated a decrease in the values of basic specific mass and saturated apparent specific mass from the base to the top of the tree, as well as from the pith to the bark. On the other hand, the moisture content presented no statistical changes in the analyzed plans. The models obtained to describe the basic specific mass and the saturated specific mass in the radial direction, despite presenting a decreasing trend, cannot be used for all axial positions because, according to the variable Dummy analyzed, they showed distinct decreasing patterns. Eugenia rostrifolia wood can be classified as moderately heavy according to the value of basic specific mass equal to $0.694 \mathrm{~g} \mathrm{~cm}^{-3}$. The physical characteristics obtained for this species showed that it has potential for use in several segments such as, for example, civil construction and furniture.
\end{abstract}


Introdução

O Brasil é um país que abriga seis biomas: Amazônia, Cerrado, Mata Atlântica, Caatinga, Pampa e Pantanal (Faria 2016). Em razão disso, possui uma ampla variedade de espécies com, aproximadamente, 516 milhões de hectares (60,7\% do seu território) de povoamentos naturais e plantados que, conforme Abraf (2010), representam a segunda maior área de florestas do mundo.

Dentre as variedades, no bioma mata atlântica, encontra-se predominantemente a família Myrtaceae, que abrange espécies relevantes no quesito da reposição vegetal (Pirola 2013). Um exemplo é a Eugenia rostrifolia, conhecida popularmente como Batinga, nativa nos estados de Santa Catarina, Rio Grande do Sul, além da Província de Missiones (Argentina), sendo endêmica do Brasil (Sobral et al. 2013).

A Eugenia rostrifolia é uma árvore de até 15 $\mathrm{m}$ de altura, com troncos retos, cilíndricos, de até 60 cm de diâmetro, abundante em matas primárias de terrenos pedregosos (Marchiori e Santos 2010). Entretanto, apesar do vasto conhecimento da fisiologia dessa planta, quando se trata de suas propriedades tecnológicas madeireiras como as físicas, por exemplo, a abordagem é escassa, levando a escassez de aplicações e possibilidades de uso racional desta nos mais variados segmentos.

Existem distinções das propriedades físicas entre e dentre espécies ou, até mesmo, em um mesmo indivíduo, as quais podem ser influenciadas principalmente pela constituição anatômica (Almeida et al. 2014). De acordo com o mesmo autor, a ausência de informações sobre os parâmetros de espécies ainda não caracterizadas, refletem em problemas técnicos encontrados ao utilizar tais madeiras. Com isso, a análise dos atributos tecnológicos do material é importante para a caracterização e destinação correta, principalmente se tratando de madeiras provenientes de espécies ainda não divulgadas na literatura.

Dentre os parâmetros influenciáveis nas propriedades físicas, aponta-se a massa específica como sendo um dos principais (Dias 2017; Zaque et al. 2018). Essa característica é compreendida por correlacionar-se com outras particularidades e também com a composição celular da madeira, expressando seu comportamento nos processos tecnológicos e industriais (Bonduelle et al. 2015).

Outro fator relevante para obtenção de um produto final com características desejáveis é o teor de umidade (Batista et al. 2011), pois a modificação desse parâmetro e sua variação no tronco das peças é fundamental para o desempenho e utilização final (Oliveira et al. 2005). Da mesma forma, o estudo da relação entre o teor de umidade e a massa específica é interessante, visto que, essas propriedades são inversamente proporcionais, pois quanto maior a quantidade de água presente na madeira, maior o volume da peça e menor a porcentagem de elementos químicos, tais como celulose, hemicelulose e lignina (Silveira et al. 2013).

Neste contexto, o objetivo do trabalho foi caracterizar as propriedades físicas de massa específica básica, massa específica aparente saturada e teor de umidade da espécie Eugenia rostrifolia ao longo do fuste e nas diferentes posições entre a medula e a casca.

\section{Material e Métodos}

Para a condução do estudo foram utilizadas três árvores com, aproximadamente, 20 anos de idade, da espécie Eugenia rostrifolia D.Legrand. conhecida popularmente como Batinga. Os indivíduos foram provenientes da floresta estadual decidual do Rio Grande do Sul localizada no município de Frederico Westphalen - RS, correspondente a uma latitude de $27^{\circ} 21^{\prime} 33^{\prime}$ ' Sul, longitude de $53^{\circ} 23^{\prime} 40^{\prime}$ ' Oeste e altitude de $566 \mathrm{~m}$ (Renz et al., 2014). De acordo com a classificação de Köppen o clima da região é do tipo $\mathrm{Cfb}$ possuindo temperatura média de $18{ }^{\circ} \mathrm{C}$ o que favorece o desenvolvimento das diversas espécies florestais.

A partir da identificação, corte e amostragem das árvores realizaram-se os procedimentos para caracterização das propriedades físicas em que, de cada indivíduo selecionado, foram seccionados discos das posições da base $(0,10 \mathrm{~m})$, no diâmetro à $1,30 \mathrm{~m}$ de altura do solo (DAP), bem como na altura comercial de $25,50,75$ e $100 \%$ do primeiro galho vivo.

Para o transporte do material até o Laboratório de Tecnologia de Produtos Florestais da Universidade Federal de Santa Maria campus Frederico Westphalen - UFSM/FW foram utilizadas embalagens plásticas que minimizaram as alterações de umidade do material. Após a identificação das amostras deu-se início ao processo de saturação total das fibras, até peso constante, por meio da submersão em água.

As propriedades físicas de massa específica e teor de umidade no sentido base-topo (longitudinal) foram determinadas a partir da confecção de cunhas simetricamente opostas provenientes dos discos, as quais continham a medula e também partes do cerne e alburno. Para o sentido radial (medula-casca) foram marcadas e seccionadas baguetas, em cada posição amostrada, as quais, após identificadas e mensuradas, foram transformadas em corpos de prova com dimensões de $1,0 \times 1,0 \mathrm{~cm}$. A posição relativa foi então obtida pela relação da distância até o centro de cada corpo de prova a partir da medula e a dimensão total da bagueta em porcentagem.

A massa específica básica (Equação 1) foi expressa pela relação entre o peso seco a $0 \%$, obtido por meio de estufa com circulação de ar forçada a uma temperatura de $103{ }^{\circ} \mathrm{C}$ até completa estabilização; e o volume verde, mensurado pelo método da balança hidrostática (Vital 1984). Seus 
resultados foram classificados de acordo com o proposto por Carvalho (1996).

$\mathrm{Meb}=\frac{\mathrm{P} 0 \%}{\mathrm{Vv}}$

Onde: $\mathrm{MEb}=$ Massa específica básica $\left(\mathrm{g} \mathrm{cm}^{-3}\right) ; \mathrm{P}_{0 \%}$ $=$ peso seco a $0 \%(\mathrm{~g}) ; \mathrm{V}_{\mathrm{V}}=$ Volume verde $\left(\mathrm{cm}^{3}\right)$.

A massa específica aparente saturada também foi determinada pela relação entre o peso e volume, entretanto ambos foram obtidos a partir da total saturação das fibras, sendo definida pela Equação 2:

$$
\text { MEas }=\frac{\text { Psat }}{\text { Vsat }}
$$

Onde: MEas $=$ Massa específica aparente na condição saturada $\left(\mathrm{g} \mathrm{cm}^{-3}\right) ; \mathrm{P}_{\text {sat }}=$ Peso na condição saturada $(\mathrm{g}) ; \mathrm{V}_{\mathrm{sat}}=$ Volume na condição saturada $\left(\mathrm{cm}^{3}\right)$.

$\mathrm{Tu}=\frac{\mathrm{Pu}-\mathrm{P}_{0 \%}}{\mathrm{P}_{0 \%}} \times 100$

Onde: $\mathrm{Tu}=$ Teor de umidade saturado $(\%) ; \mathrm{P}_{\mathrm{u}}=$ Peso saturado $(\mathrm{g}) ; \mathrm{P}_{0 \%}=$ Peso seco a $0 \%(\mathrm{~g})$.

Os dados das propriedades físicas amostradas foram plotados em função das posições relativas estudadas (longitudinal e radial) e submetidos à análise de regressão utilizando o pacote Statistical Analysis System, no qual foi aplicado o procedimento Stepwise de modelagem de regressão (Equação 4).

MEb, MEas, Tu $=\mathrm{f}\left(\mathrm{P}, 1 / \mathrm{P}, \mathrm{P}^{2}, 1 / \mathrm{P}^{2}, \log (\mathrm{P}), 1 / \operatorname{Ln}(\mathrm{P})\right)$ (4)

Onde: $\mathrm{MEb}=$ Massa específica básica $\left(\mathrm{g} \mathrm{cm}^{-3}\right)$; MEas $=$ Massa específica aparente saturada $\left(\mathrm{g} \mathrm{cm}^{-3}\right)$;
$\mathrm{Tu}=$ Teor de umidade saturado (\%); $\mathrm{P}=$ Posição relativa longitudinal e radial $(\%)$.

O modelo utilizado foi selecionado com base no $\mathrm{R}^{2}$ aj. (Coeficiente de determinação), Syx (Erro padrão da estimativa), F calc. (Valor de F calculado para o modelo) e análise gráfica dos resíduos.

Em seguida, em cada modelo escolhido para descrever as características físicas analisadas no sentido radial, foram adicionadas variáveis Dummy, definidas pelas posições relativas axiais, a fim de verificar a variação dos parâmetros tecnológicos ao longo do fuste (Equação 5).

$$
\text { MEb, MEas, } T u=f(X ; D ; X . D)
$$

Onde: $\mathrm{MEb}=$ Massa específica básica $\left(\mathrm{g} \mathrm{cm}^{-3}\right)$; MEas = Massa específica aparente saturada $\left(\mathrm{g} \mathrm{cm}^{-3}\right)$; $\mathrm{Tu}=$ Teor de umidade saturado $(\%) ; \mathrm{X}=$ Posição relativa (sentido medula-casca) selecionada pelo procedimento Stepwise de regressão (\%); D = Variável Dummy correspondente à posição axial; X.D = Interação de X com a variável Dummy.

\section{Resultados}

A massa específica básica e aparente saturada apresentaram valores médios iguais a 0,694 e 1,235 $\mathrm{g} \mathrm{cm}^{-3}$, respectivamente. Com esses resultados, também foi possível quantificar a massa seca e úmida para cada indivíduo, sendo obtidas médias de 403,1 e $715,7 \mathrm{~kg}$, respectivamente.

Os valores médios para as propriedades estudadas em cada posição do sentido longitudinal das árvores de Eugenia rostrifolia demonstraram uma tendência de decréscimo da base para o topo para as massas específicas básica e aparente saturada, enquanto o teor de umidade apresentou um acréscimo ao longo do fuste (Tabela 1).

Tabela 1. Massa específica básica, aparente saturada e teor de umidade médios por posição no sentido base-topo da espécie Eugenia rostrifolia.

\begin{tabular}{cccc}
\hline Posição & $\begin{array}{c}\text { Massa específica } \\
\text { básica }\left(\mathbf{g ~ c m}^{-3}\right)\end{array}$ & $\begin{array}{c}\text { Massa específica aparente } \\
\text { saturada }\left(\mathbf{g ~ c m}^{-3}\right)\end{array}$ & $\begin{array}{c}\text { Teor de } \\
\text { umidade }(\%)\end{array}$ \\
\hline $0,1 \mathrm{~m}$ & $0,732 \pm 0,054$ & $1,257 \pm 0,014$ & $71,5 \pm 16,1$ \\
DAP & $0,693 \pm 0,053$ & $1,241 \pm 0,002$ & $70,9 \pm 14,4$ \\
$25 \%$ & $0,714 \pm 0,007$ & $1,237 \pm 0,011$ & $73,3 \pm 3,02$ \\
$50 \%$ & $0,683 \pm 0,017$ & $1,224 \pm 0,017$ & $79,4 \pm 2,45$ \\
$75 \%$ & $0,677 \pm 0,013$ & $1,229 \pm 0,003$ & $81,6 \pm 3,60$ \\
$100 \%$ & $0,677 \pm 0,033$ & $1,228 \pm 0,013$ & $84,4 \pm 9,86$ \\
\hline
\end{tabular}

Onde: DAP = diâmetro à altura de 1,30 $\mathrm{m}$ do solo. Valores entre parênteses indicam o desvio padrão.

Os coeficientes e as estatísticas da seleção das equações para descrever a massa específica básica e a aparente saturada das árvores de Eugenia rostrifolia em função da posição relativa, nos 
sentidos base-topo e medula-casca, encontram-se na Tabela 2. A adição de mais variáveis independentes aos modelos não foi possível para um nível de $5 \%$ de probabilidade de erro. Cabe ressaltar ainda que foi observada uma tendência de acréscimo para o teor de umidade, tanto no sentido base-topo quanto no medula-casca, porém sem diferença estatística.

Tabela 2. Modelos de regressão obtidos pelo procedimento Stepwise para descrever massa específica básica e a aparente saturada de Eugenia rostrifolia em função da posição relativa no sentido base-topo e medula-casca.

\begin{tabular}{|c|c|c|c|c|c|c|c|c|}
\hline \multirow{2}{*}{ Posição } & \multirow{2}{*}{ Passo } & \multirow{2}{*}{ Modelo } & \multicolumn{4}{|c|}{ Estatísticas } & \multirow{2}{*}{$\mathbf{R}^{2}{ }_{\text {aj. }}$} & \multirow{2}{*}{$\mathbf{F}$} \\
\hline & & & $\mathbf{b}_{\mathbf{j}}$ & Valor & $F^{\prime}$ & Prob $>$ F & & \\
\hline \multirow{2}{*}{ Axial } & \multirow{2}{*}{1} & \multirow{2}{*}{$\mathrm{MEb}=\mathrm{b}_{0}+\mathrm{b}_{1}\left(\mathrm{P}^{2}\right)$} & $\mathrm{b}_{0}$ & 0,725 & 41187,4 & $<0,001 * *$ & \multirow{2}{*}{0,0137} & \multirow{2}{*}{4,1} \\
\hline & & & $b_{1}$ & $-0,0000017$ & 4,05 & $0,0455^{*}$ & & \\
\hline \multirow{2}{*}{ Axial } & \multirow{2}{*}{1} & \multirow{2}{*}{ MEas $=b_{0}+b_{1}(P)$} & $\mathrm{b}_{0}$ & 1,254 & 282767 & $<0,001 * *$ & \multirow{2}{*}{0,0624} & \multirow{2}{*}{15,6} \\
\hline & & & $\mathrm{b}_{1}$ & $-0,0001826$ & 15,64 & $<0,001 * *$ & & \\
\hline \multirow{2}{*}{ Radial } & \multirow{2}{*}{1} & \multirow{2}{*}{$\mathrm{MEb}=\mathrm{b}_{0}+\mathrm{b}_{1}(1 / \mathrm{P})$} & $\mathrm{b}_{0}$ & 0,711 & 26417,8 & $<0,001 * *$ & \multirow{2}{*}{0,0254} & \multirow{2}{*}{6,7} \\
\hline & & & $b_{1}$ & 0,338 & 6,72 & $0,0102 *$ & & \\
\hline \multirow{2}{*}{ Radial } & \multirow{2}{*}{1} & \multirow{2}{*}{ MEas $=b_{0}+b_{1}(1 / L n P)$} & $\mathrm{b}_{0}$ & 1,225 & 23937,9 & $<0,001 * *$ & \multirow{2}{*}{0,0288} & \multirow{2}{*}{7,5} \\
\hline & & & $\mathrm{b}_{1}$ & 0,0803 & 7,52 & $0,0066 * *$ & & \\
\hline
\end{tabular}

Onde: $\mathrm{MEb}=$ massa específica básica $\left(\mathrm{g} \mathrm{cm}^{-3}\right) ;$ MEas = massa específica aparente saturada $\left(\mathrm{g} \mathrm{cm}^{-3}\right) ; \mathrm{P}=$ posição relativa no sentido axial (base-topo) e radial (medula-casca) (\%); b"i" = parâmetros da equação; F' = valor de $\mathrm{F}$ calculado para teste de hipótese dos parâmetros; Prob. $>\mathrm{F}=$ nível de probabilidade de erro; R2aj. = coeficiente de determinação ajustado; F = valor de F calculado para o modelo; ** = significativo ao nível de $1 \%$ de probabilidade de erro; * significativo ao nível de $5 \%$ de probabilidade de erro.

As massas específicas básicas e aparente saturada evidenciaram variações semelhantes no sentido longitudinal, sendo maiores próximas da base e reduzindo ao longo do fuste. Já para o sentido
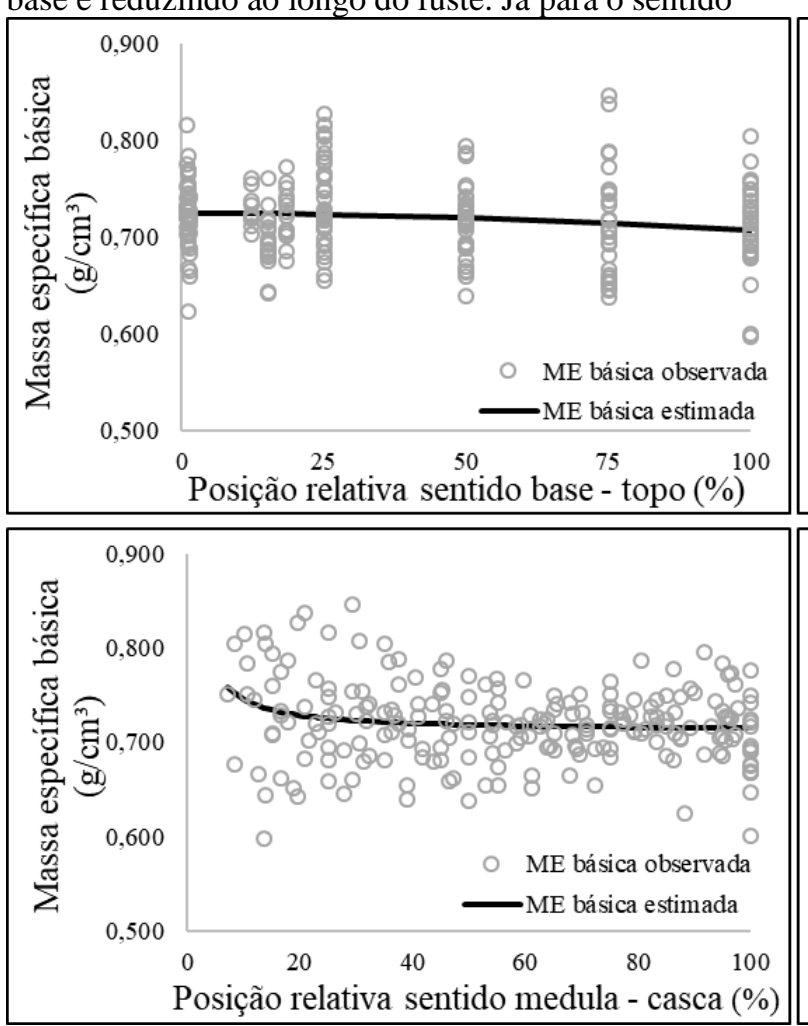

radial, essas características físicas apresentaram os maiores valores próximos à medula com tendência de diminuição em direção à casca.
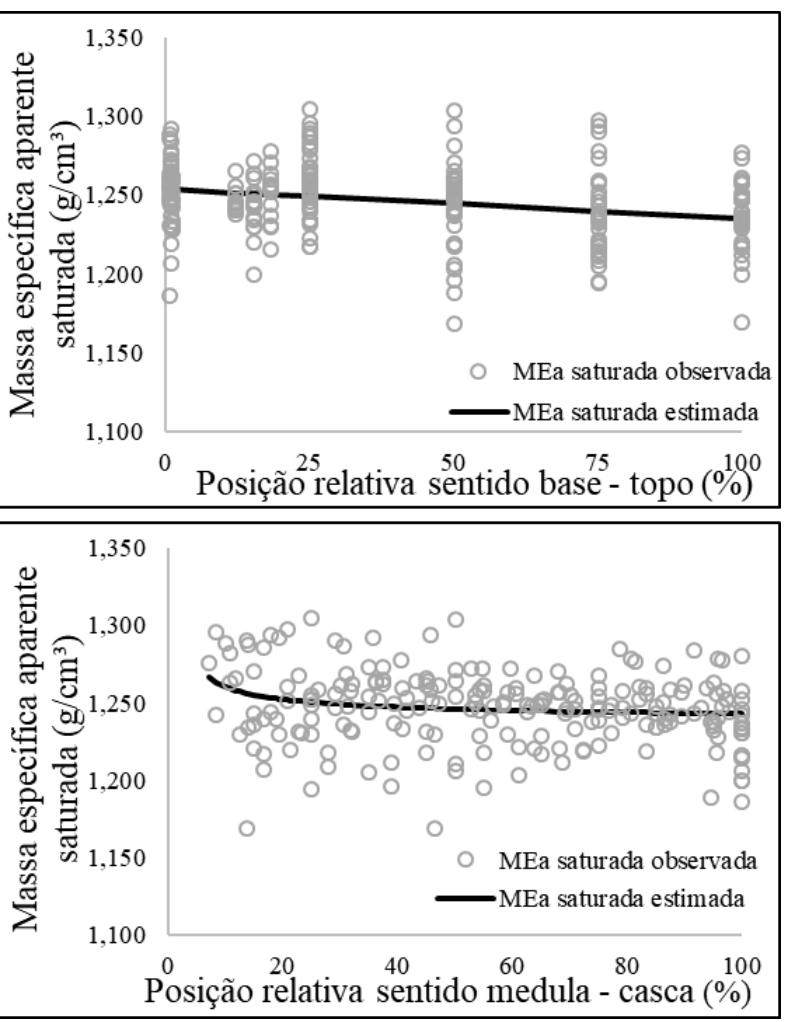

Figura 1. Variação longitudinal e radial das massas específicas básica e aparente saturada de Eugenia rostrifolia em função da posição relativa. 
Nas equações selecionadas para descrever a massa específica básica e aparente saturada, os dados em função da posição relativa no sentido medula- casca foram relacionados com as respectivas posições axiais e submetidos à análise de regressão com o emprego de variável Dummy (Tabela 3).

Tabela 3. Análise de regressão com variável Dummy (tipo SS1) da massa específica básica e massa específica aparente saturada de Eugenia rostrifolia em função da posição relativa no sentido medula-casca ao longo do fuste.

Massa específica básica

\begin{tabular}{cc}
\hline FV & QM \\
\hline Modelo & $0,00531101^{* *}$ \\
1/P & $0,01172182^{* *}$ \\
D1 & $0,00124056^{\text {ns }}$ \\
D2 & $0,00213068^{\text {ns }}$ \\
D3 & $0,01921270^{* *}$ \\
D4 & $0,00031784^{\text {ns }}$ \\
D5 & $0,00000677^{\text {ns }}$ \\
D6 &.
\end{tabular}

Massa específica aparente saturada

\begin{tabular}{cc}
\hline FV & QM \\
\hline Modelo & $0,00208327^{* *}$ \\
1/LnP & $0,00415176^{* *}$ \\
D1 & $0,00142921^{\text {ns }}$ \\
D2 & $0,00002652^{\text {ns }}$ \\
D3 & $0,01037490^{* *}$ \\
D4 & $0,00058342^{\text {ns }}$ \\
D5 & $0,00007013^{\text {ns }}$ \\
D6 & $\cdot$ \\
D1. 1/LnP & $0,00002711^{\text {ns }}$ \\
D2. 1/LnP & $0,00085396^{\text {ns }}$ \\
D3. 1/LnP & $0,00127832^{\text {ns }}$ \\
D4. 1/LnP & $0,00408423^{* *}$ \\
D5. 1/LnP & $0,00003638^{\text {ns }}$ \\
D6. 1/LnP & $\cdot$ \\
Erro & 0,00048814
\end{tabular}

Onde: $\mathrm{FV}=$ fonte de variação; $\mathrm{QM}=$ quadrado médio; $\mathrm{P}=$ posição relativa no sentido medula-casca (\%); $\mathrm{D}$ 'i” $=$ posição relativa no sentido base-topo (Dummy); $\mathrm{D}$ י’1/P = interação posição relativa no sentido base-topo (Dummy) com a variável 1/P; $\mathrm{D}$ יi $1 / \mathrm{LnP}$ = interação posição relativa no sentido base-topo (Dummy) com a variável 1/LnP; ** = significativo ao nível de $1 \%$ de probabilidade de erro; ${ }^{*}=$ significativo ao nível de $5 \%$ de probabilidade de erro; ns = não significativo ao nível de $5 \%$ de probabilidade de erro.

Com base na Tabela 3, verificou-se que os modelos obtidos para descrever a massa específica básica e a aparente saturada no sentido radial não podem ser utilizados para todas as posições axiais, pois existem variáveis Dummy, bem como interações, empregadas na equação, que foram significativas em nível máximo de $\alpha=5 \%$ de probabilidade de erro.

Dessa forma, a massa específica básica e aparente saturada em função da posição relativa no sentido radial utilizando variável Dummy como posição axial, pelas equações reajustadas descritas abaixo, podem ser observadas na Figura 2.

$\mathrm{MEb}=0,7074+0,3992(1 / \mathrm{P})+0,0218 \mathrm{D} 3-$ $0,3942 \mathrm{D} 2 \cdot 1 / \mathrm{P}+0,1874 \mathrm{D} 4.1 / \mathrm{P}$
MEas $=1,2233+0,0786(1 / \mathrm{LnP})+0,0147 \mathrm{D} 3-$ $0,00054 \mathrm{D} 4.1 / \mathrm{LnP}$

Onde: $\mathrm{MEb}=$ Massa específica básica $\left(\mathrm{g} \mathrm{cm}^{-}\right.$ $\left.{ }^{3}\right)$; MEas = Massa específica aparente saturada $\left(\mathrm{g} \mathrm{cm}^{-}\right.$ ${ }^{3}$ ); 1/P e 1/LnP = Posições relativas (sentido medulacasca) selecionadas pelo procedimento Stepwise de regressão (\%); D3 = Variável Dummy correspondente à posição axial de $25 \%$ de altura do primeiro galho vivo; D2.1/P, D4.1/P e D4.1/LnP = Interações da variável Dummy correspondente à posição axial do diâmetro à altura de $1,30 \mathrm{~m}$ do solo (D2) e 50\% de altura do primeiro galho vivo (D4) com as posições selecionadas pelo procedimento Stepwise de regressão, respectivamente. 

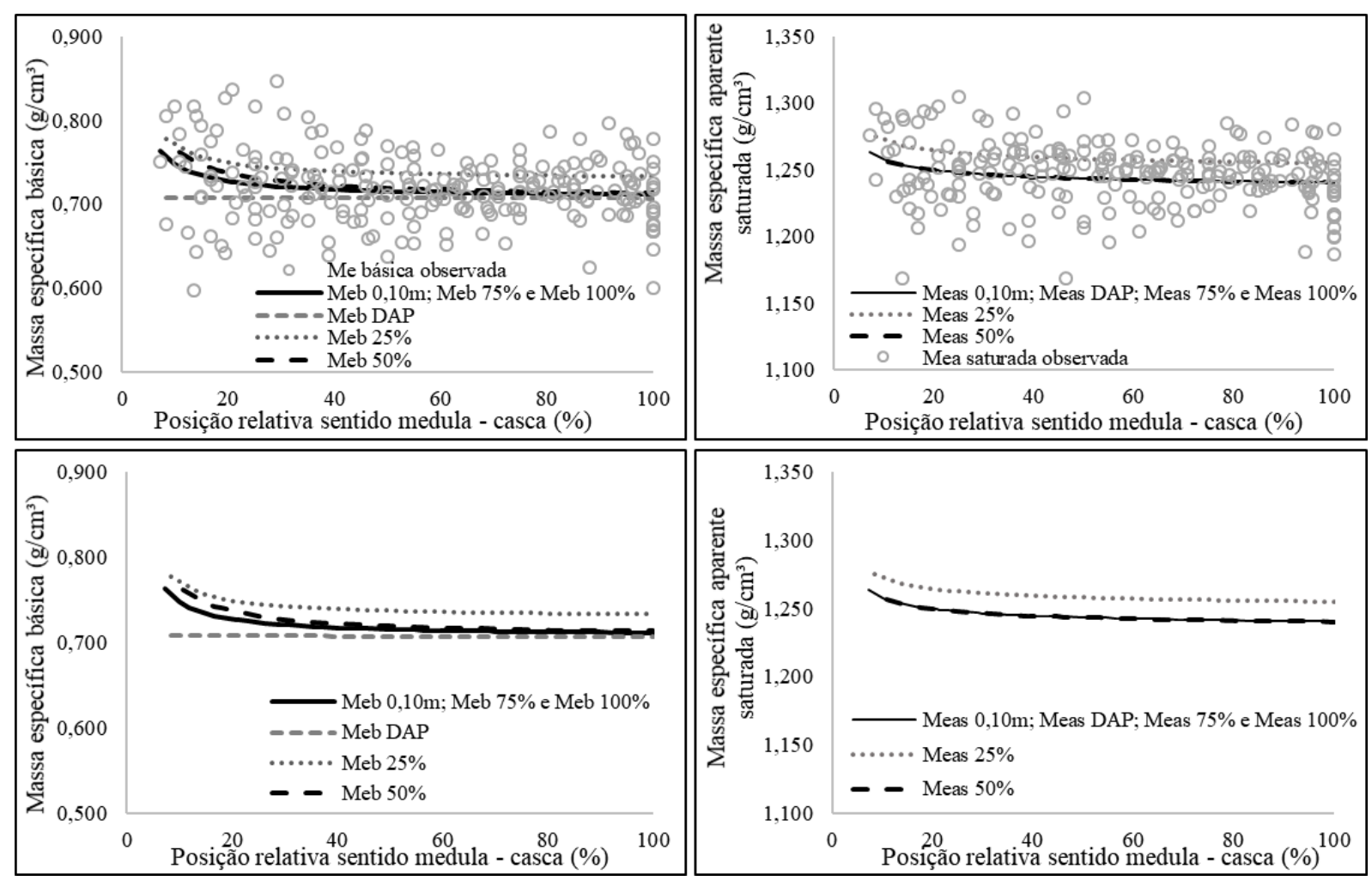

Figura 2. Variação das massas específicas básica e aparente saturada de Eugenia rostrifolia em função da posição relativa no sentido medula-casca ao longo do fuste.

\section{Discussão}

The selectivity in the pulping process may be A madeira de Eugenia rostrifolia pode ser classificada como moderadamente pesada pois, de acordo com a classe proposta por Carvalho (1996), apresentou um valor de massa específica básica que se enquadrou na amplitude de 0,650 a $0,790 \mathrm{~g} \mathrm{~cm}^{-3}$.

De acordo com a Figura 1, a massa específica básica, bem como, a massa específica aparente saturada, tiveram padrões semelhantes de variação no sentido radial, com valores decrescentes em direção à casca. Os resultados encontrados estão em conformidade com a literatura para outras espécies arbóreas folhosas que também evidenciaram essa tendência no sentido medula-casca, conforme abordado por Gatto et al. (2012) para Carya illinoinensis proveniente das regiões Nordeste e Depressão Central do estado do Rio Grande do Sul. Essa redução da massa específica pode ser resultado do tamanho das paredes celulares das fibras ou a diferença de proporção entre a espessura das fibras e dos vasos (Nisgoski et al. 2011).

No sentido longitudinal, a massa específica básica e a massa específica aparente saturada apresentaram comportamento decrescente com valores maiores na base e reduzindo ao longo do fuste. Esses resultados concordaram com o estudo de Machado et al. (2014), onde os autores notaram valores menores de massa específica aparente a $12 \%$ no topo da árvore, correspondendo a um material mais jovem, e maiores na base, representando o lenho adulto, para a madeira de Acacia melanoxylon.
Esses pesquisadores discutiram ainda que, o comportamento da massa específica na base do tronco possui relação com a idade e o sistema radicular das árvores estudadas; variáveis essas que refletem nos dados maiores ou menores dessa propriedade.

Os dados obtidos no sentido base-topo também corroboraram com o comportamento observado por Bonduelle et al. (2015) para a madeira de Tectona grandis onde ocorreu um decréscimo da massa específica básica da base ao meio do tronco. A variação no sentido base-topo das árvores não possui padrões definidos de acordo com Eloy et al. (2013), contudo, o mais comumente observado é uma redução dos valores ao longo do fuste.

Para outras espécies da mesma família da Eugenia rostrifolia, Beltrame et al. (2015) estudando clones de Eucalipto, observaram a redução da massa específica no sentido base-topo, corraborando com os resultados obtidos no presente trabalho. Esse comportamento também foi observado para outras espécies nativas do Rio Grande do Sul, como a Cordia americana e Alchornea triplinervia estudadas por Trautenmüller et al. (2014), no qual os valores de massa específica foram decrescentes da base para o topo. O mesmo foi aferido por Candaten et al. (2018) para a madeira de Maclura tinctoria, outra nativa do Rio Grande do Sul, onde a massa específica básica e massa específica aparente saturada reduziram no sentido longitudinal.

Os resultados para massa específica básica e aparente saturada podem ser relacionados com o teor 
de umidade. Assim, no caso de redução da massa específica básica tem-se um aumento do teor de umidade e, de modo contrário, para a massa específica aparente saturada. Esses comportamentos também foram observados por Juizo et al. (2015), Friederichs et al. (2015) e Candaten et al. (2018), onde nos maiores picos de massa específica observou-se menores teores de umidade, e viceversa. Ocorrência essa que, conforme discutido pelos autores, está relacionada com o ponto de saturação das fibras da madeira, que em situações acima de $30 \%$ de umidade, apenas a massa é alterada, não apresentando mais variações no seu volume, implicando diretamente nos resultados obtidos para a massa específica da madeira, independente da espécie.

A comparação de resultados das características físicas de massa específica básica, aparente saturada e teor de umidade, com a espécie de Eugenia rostrifolia não foi possível em função da ausência de literatura a respeito dessas variáveis para a espécie, fundamentando a importância desse estudo e tornando disponível essas informações para trabalhos futuros.

\section{Conclusões}

A massa específica básica e aparente saturada do fuste de Eugenia rostrifolia apresentaram um decréscimo da base para o topo da árvore, bem como da medula até a casca. Já o teor de umidade, apesar de tendência crescente no sentido axial, não evidenciou alterações estatísticas nos planos analisados.

Os modelos para descrever a massa específica básica e a massa específica aparente saturada no sentido radial, apesar de tendência de decréscimo, não podem ser utilizados para todas as posições axiais, pois apresentam padrões decrescentes distintos.

A madeira de Eugenia rostrifolia pode ser classificada como moderadamente pesada demonstrando que a mesma tem potencial para uso em vários segmentos como, por exemplo, construção civil e movelaria.

\section{Referências}

Associação Brasileira de Florestas Plantadas ABRAF. Anuário estatístico da ABRAF 2010 ano base 2009, p, 140, Brasília 2010.

Almeida DH, Molina JC, Almeida TH, Calil Junior, C, Alves ACL (2014) Determinação do teor de umidade e densidade básica pra espécies de Pinus e Eucalipto. Revista Científica Eletrônica da FAIT.

American Society for Testing and Materials. ASTM D 143- 94: standard test methods for small clear specimens of timber. Philadelphia, 1995.
Batista WR, Guarneti JE, Severo D, Klar AE (2011) Calibração de um equipamento TDR (reflectometria por domínio do tempo) para a determinação da umidade da madeira de Grevillea robusta (Cunn.). Revista Energia na Agricultura, 26 (2): 129-145. doi.org/10.17224/EnergAgric.2011v26n2p129-145.

Beltrame R, Peres ML, Delucis RA, Freitas DL, Gatto DA, Haselein CR (2015) Tensões de crescimento longitudinais e propriedades mecânicas da madeira de clones de Eucalyptus spp. Revista Matéria, $20 \quad$ (4): $1061 \quad$ - 1074. http://dx.doi.org/10.1590/S1517-

707620150004.0108 .

Bonduelle GM, Iwakiri S, Trianoski R, Prata JG, Rocha VY (2015) Análise da massa específica e da retratibilidade da madeira de Tectona grandis nos sentidos axial e radial do tronco. Revista Floresta, 45 (4): 971-680. http://dx.doi.org/10.5380/rf.v45i4.31991.

Candaten L, Mangini, TS, Bandera E, Costa HWD, Trevisan R (2018) Relação da massa específica e teor de umidade da madeira de Maclura tinctoria ao longo do fuste. Enciclopédia Biosfera, 15 (28): 845854. 10.18677/EnciBio_2018B68.

Carvalho, A. Madeiras portuguesas: estrutura anatômica, propriedades, utilizações. Lisboa: Instituto Florestal, 1996. v. 1. 340 p. Disponível em: <http://www.estgv.ipv.pt/PaginasPessoais/jqomarce lo/Tim3/tim3_TP1_Na2.pdf >. Acesso em: 27 de maio de 2019.

Dias DC, Colodette JL, Thiersch CR, Leite HG, Gomide JL (2017) Uso da técnica de resistografia e de variáveis dendrométricas na modelagem da densidade básica de povoamentos clonais de Eucalyptus. Revista Ciência Florestal, 27(2): 609619. http://dx.doi.org/10.5902/1980509827746.

Eloy E, Caron BO, Trevisan R, Schimdt D, Zanon MLB, Behling A, Monteiro GC (2013) Variação longitudinal e efeito do espaçamento na massa específica básica da madeira de Mimosa scabrella e Ateleia glazioveana. Floresta, 43 (2): 327 -334.

Faria, CO (2016) Governança da biodiversidade sob a perspectiva de sistemas sócio-ecológicos: o caso do bioma mata atlântica. . . Tese (Doutorado em Planejamento Energético) - Universidade Federal do Rio de Janeiro, Rio de Janeiro. 126f.

Friederichs G, Brand MA, Carvalho AF, Küster LC (2015) Qualidade da madeira e do carvão vegetal de 
bracatinga (Mimosa scabrella Benth.). Ciência da Madeira, 6 (2): 79-87, 2015. 10.12953/21776830/rcm.v6n2p79-8.

Gatto DA, Martins MF, Cademartori PHG, Stangerlin DM, Calegari L, Beltrame R (2012) Segregação do lenho de nogueira-pecã (Carya illinoinensis) pela variação radial da massa específica básica. Revista Brasileira de Ciências Agrárias, 7: 838-843. 10.5039/agraria.v7isa1935.

Juizo CGF, Loiola PL, Zen LR, Marchesan R, Carvalho DE, Bila NF, Egas A, Rocha MP, Klitzke RJ (2015) Variação radial das propriedades físicas da madeira de Pinus patula plantados em Moçambique. Pesquisa Florestal Brasileira 35 (83): 285-292.

https://doi.org/10.4336/2015.pfb.35.83.771.

Machado JS, Louzada J, Santos AJ, Nunes L, Anjos O, Rodrigues J, Simoes R, Pereira H (2014) Variation of wood density and mechanical properties of blackwood (Acacia melanoxylon R. Br.). Materials and Design, 56: 975-980, 2014. https://doi.org/10.1016/j.matdes.2013.12.016.

Marchiori JNC, Santos SR (2010) Anatomia da madeira de duas espécies de Eugenia L. (Myrtaceae). Balduinia, 21: 15-21. http://dx.doi.org/10.5902/2358198014099.

Nisgoski S, Trianoski R, Muniz GIB, Matos JLM, Batista FRR (2011) Anatomia da madeira de Toona ciliata características das fibras para produção de papel. Revista Floresta, 41 (4): 717-728, 2011.

Oliveira JTS, Hellmeister JC, Tomazello Filho M (2005) Variação do teor de umidade e da densidade básica na madeira de sete espécies de eucalipto. Revista Árvore, 29 (1): 115-127, 2005. https://doi.org/10.1590/S010067622005000100013.

Pirola, K (2013) Caracterização fisiológica e conservação de sementes de oito fruteiras nativas do Bioma Floresta com Araucária. Dissertação. (Mestrado em Agronomia. Universidade Tecnológica Federal do Paraná, Pato Branco. 129f.

Renz VD, Ritter LG, Mancuso MA, Hass A, Conceição SR, Garlet G (2014) Delimitação e monitoramento vegetativo da bacia hidrográfica do Lajeado Chiquinha. REGET/UFSM, v. 18, n. 1, p. 172-177, 2014. http://dx.doi.org/10.5902/2236117011103.

Sobral, M (2013) Myrtaceae in Lista de Espécies da Flora do Brasil. Jardim Botânico do Rio de Janeiro.

Silveira LHC, Rezende AV, Vale AT (2013) Teor de umidade e densidade básica da madeira de nove espécies comerciais amazônicas. Acta Amazônica,
Brasília. https://doi.org/10.1590/S004459672013000200007.

Trautenmüller JW, Balbinot R, Borella J, Trevisan R, Balestrin D, Vendruscolo R, Sabadini AM (2014) Variação longitudinal da massa específica básica da madeira de Cordia americana e Alchornea triplinervia. Ciência Rural, 44 (5): 817-821.

Vital, B. R (1984) Métodos de determinação da densidade da madeira. p. 24. (Boletim Técnico, 2).

Zaque LAM, Ferreira MD, Melo RR (2018) Variação radial e longitudinal da massa específica básica da madeira de Araucaria angustifolia com diferentes idades. Pesquisa Florestal Brasileira, 38: 1-5. 10.4336/2018.pfb.38e201601368. 